\title{
END FIELD STRUCTURES FOR LINEAR/HELICAL INSERTION DEVICES.
}

\author{
J. Chavanne, P. Elleaume, P. Van Vaerenbergh \\ ESRF, B.P. 220, F-38043 GRENOBLE Cedex France
}

\begin{abstract}
The field integral tolerances required on third generation synchrotron sources are of the order of $10-20 \mathrm{Gcm}$ in the whole gap range of an insertion device. This can be achieved without electromagnet correction by a proper magnetic design of the field termination. The paper describes several such end field terminations to be used for planar undulators. A new termination valid for an APPLE II undulator is presented which produces a field integral variation smaller than $20 \mathrm{Gcm}$ for any useful setting of the magnetic gap and phase. It compares quite favorably with other known type of terminations.
\end{abstract}

\section{INTRODUCTION}

An ideal insertion device must not perturb the closed orbit and the dynamics of the electron beam in a storage ring for any value of the magnetic gap. The field integral is defined as a function of the transverse coordinates $\mathrm{x}$ and $\mathrm{z}$ as :

$$
I(x, z)=\int_{-\infty}^{\infty} B(x, z, s) d s=\left(\begin{array}{c}
I_{x} \\
I_{z}
\end{array}\right)
$$

The derivative of the field integrals vs. $\mathrm{x}$ and $\mathrm{z}$ must also be equal to zero. The second field integral defined as:

$$
J(x, z)=\int_{-\infty}^{\infty}\left(\int_{-\infty}^{s} B\left(x, z, s^{\prime}\right) d s^{\prime}\right) d s=\left(\begin{array}{c}
J_{x} \\
J_{z}
\end{array}\right)
$$

must also be equal to zero. Two kind of magnetic errors are in general responsible for non zero field integral in real devices. The first one that we also call "random errors" come from the non-uniformity of the magnetization in the blocks and from positioning errors of the blocks. Because they are largely unpredictable, this type of errors cannot be taken into account at the design stage. They can be reduced with adequate choice of manufacturing process of the permanent magnets such as die pressing method and/or by characterizing magnetically each block and then by sorting and pairing the blocks in order to cancel the field errors introduced by each blocks. In addition, some field integral shimming [1] can be used to reduce the final errors. The second type of error that we call "systematic errors" originates from the extremities of the magnetic structure where the field periodicity is broken. Experience shows that it is difficult to efficiently correct the systematic errors using shimming and most people use correction coils (active correction). Nevertheless, systematic errors can be fully predicted and to some extent corrected using a 3D magnetostatics computer code (passive correction). In the particular case of pure permanent magnet undulators, the non unit permeability of permanent magnet materials $(\mathrm{NdFeB}, \mathrm{SmCo})$ can be responsible for a non-zero field integral generated at each extremity of the undulator. The next sections present several designs of passively corrected terminations to be used with either planar undulator or APPLE-II linear/helical undulators.

\section{PLANAR FIELD UNDULATORS}

Figure 1 presents a 3D view of a conventional termination (type A) of a pure permanent magnet undulator. The structure is terminated with a vertically magnetized half magnet.

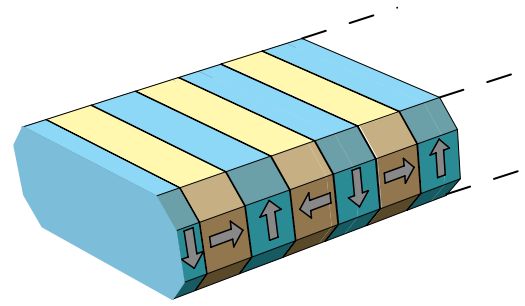

Figure 1: 3D view of a conventional termination (type A) of a pure permanent magnet undulator (lower part only).

The termination of type A shown in Figure 1 produces a significant variation of the field integral as a function of the magnetic gap (see Figure 4). This can be explained numerically by taking into account of the non unit permeability and the anisotropy of the magnetic material. From the experience made with blocks from various magnet suppliers, we have found that $\mathrm{NdFeB}$ can be magnetically described with the remanent field given by the supplier and a linear (transverse) permeability of 1.06 (1.17). Two special end field structures have been designed at the ESRF using the code RADIA [2]. The first one called " $\mathrm{B}$ " is shown on Figure 2. It is derived from type A by adding at the end an horizontally magnetized block which longitudinal dimension (along the electron beam) is approximately equal to $3 / 20$ of the undulator period $\lambda_{0}$. As shown in Figure 4, type B termination reduces the field integral variations with gap by a factor 4 to 5 . Nevertheless, contrary to type A, it does not allow the proper phasing between segmented undulators. 


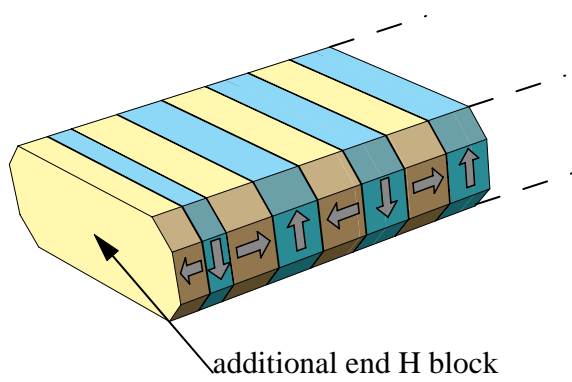

Figure $2: 3 \mathrm{D}$ view of a termination of type $\mathrm{B}$.

To satisfy both requirements of negligible field integral variation with gap and phasing between segments, we have designed and implemented the type C termination shown on Figure 3.

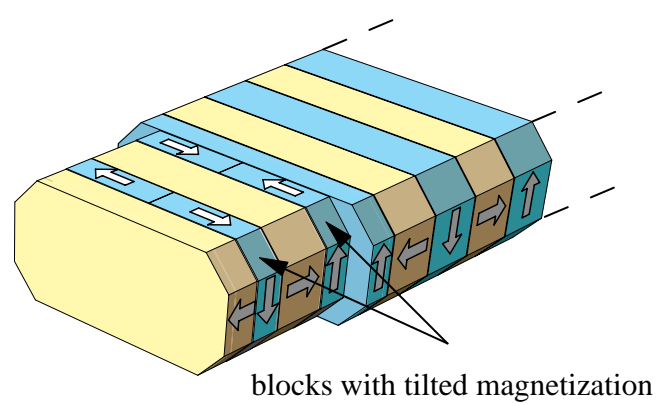

Figure 3: 3D view of $\mathrm{C}$ type termination

This termination includes a number of magnet blocks with smaller horizontal sizes, some of which have an inclined magnetization with respect to the vertical axis. This termination also presents the advantage that an air gap of $6 \mathrm{~mm}$ (for a $42 \mathrm{~mm}$ period) is left between two undulator segments while preserving the correct phasing for any useful gap value. All pure permanent magnet undulators of the ESRF include terminations of type B or $\mathrm{C}$ depending on the phasing requirements. From the reasons explained above, Type $\mathrm{C}$ structure is the preferred one. It has been implemented to undulator periods ranging from 20 to $42 \mathrm{~mm}$.

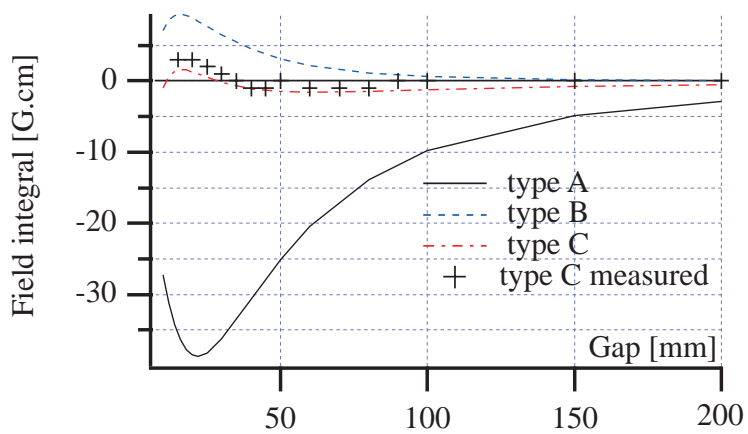

Figure 4: Vertical field integral/extremity computed for terminations of type $\mathrm{A}, \mathrm{B}$ or $\mathrm{C}$. The undulator period is $40 \mathrm{~mm}$ and the magnet are made of $\mathrm{NdFeB}$. The crosses are the field integrals measured on an existing termination.

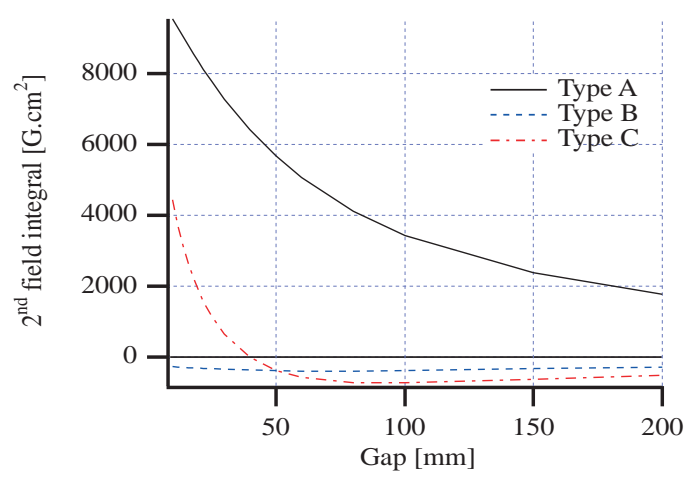

Figure 5: Second vertical field integral/extremity computed for the terminations of type A,B and C. The undulator period is $40 \mathrm{~mm}$ and the magnet are made of $\mathrm{NdFeB}$

\section{APPLE II END FIELD STRUCTURES}

Helical undulators based on the APPLE II structure [3] have been built or are under construction in a number of facilities [4],[5],[6],[7] and at the ESRF. These devices are pure permanent magnet structures. The magnet assembly is split into four magnet arrays. Two magnet arrays, one of the upper magnet arrays and one of the lower magnet arrays are simultaneously displaced longitudinally. The associated motion is called the phasing and it results in a change of the circular polarization from left to right hand side. Most APPLE II undulators built in the world are made with $\mathrm{NdFeB}$ blocks and terminated as in Figure 1. The non-unit permeability of $\mathrm{NdFeB}$ is responsible for some residual field integrals which depends on both the gap and the phase (see [8]).

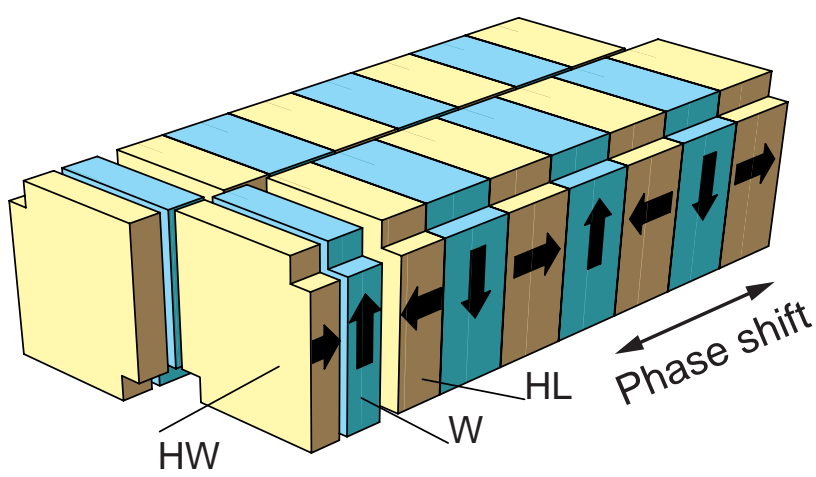

Figure 7: New end field structure for APPLE II type helical undulators.

Recently, a modified termination structure has been designed at ELETTRA for the construction of a number of APPLE II devices [6]. This type of termination has been optimized to produce displacement free trajectory and a reduced fringe field but the residual field integral is large and the operation of such a device at the ESRF would require the used of an active correction. Figure 7 presents a 
novel type of end structure which greatly reduces the field integral variations induced by both the gap and the phase. It essentially consists in three magnet blocks placed at the end with some air gaps. For a $70 \mathrm{~mm}$ period and $\mathrm{NdFeB}$ material, the normal block dimensions are 35 x $35 \times 17.5 \mathrm{~mm}$ (horizontal x vertical x longitudinal). The magnets of type HL, W and HW (See Figure 7) have the same cross-section but a different longitudinal dimension equal to $11.7,8.7$ and 7.3 (respectively). The air gap is $5 \mathrm{~mm}(2 \mathrm{~mm}$ ) between the HL and W ( W and HW) magnet blocks. The two air gaps have been optimized to reduce the vertical field integral dependence vs. phase shift. Figure 8 presents the predicted field integral vs. phase of the three type of extremities discussed above. They are all computed for a $70 \mathrm{~mm}$ period at the minimum magnetic gap of $16 \mathrm{~mm}$ assuming a symmetric (anti-symmetric) configuration of the vertical (horizontal) field components.

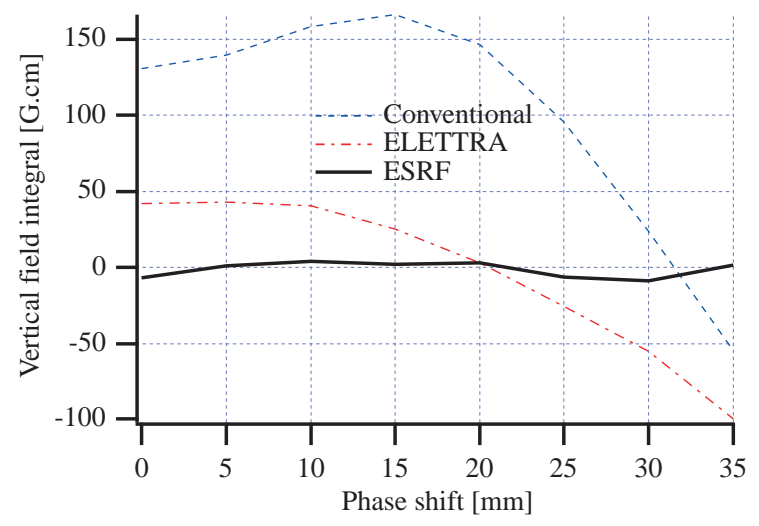

Figure 8. Vertical field integral vs. phase shift for an APPLE II undulator equipped with different field terminations. The period is $70 \mathrm{~mm}$, the gap is $16 \mathrm{~mm}$. The magnet blocks are made of $\mathrm{NdFeB}$ and the vertical field presents a symmetric configuration between each extremity.

A complete study of the field integral vs. gap and phase induced by the new termination is presented in Figure 9 and shows a maximal variation of $\pm 7 \mathrm{Gcm}$ allowing the removal of the end correction coils.

\section{CONCLUSION}

By making use of a 3D magnetostatics code, it is possible to design passively corrected terminations for the planar and APPLE II linear/helical undulator. Full designs have been given for a particular period. If one needs to design an undulator with an other period, one can scale the longitudinal and transverse dimensions to the period and obtain a reasonable correction but a short re-optimization is likely to further improve the correction. The RADIA code has been found to be quite efficient for this task. A single structure is solved and the field integrals are computed within 30 seconds, allowing a systematic and automatic investigation of a large number of magnet configurations. The ESRF ID group is presently starting the construction of several helical undulators of the APPLE II type with periods of 38 and $88 \mathrm{~mm}$. In both cases a termination similar as that of Figure 7 has been optimized which produces a maximum field integral variations with phase and gap smaller than $\pm 10 \mathrm{Gcm}$. Note that RADIA can also be used to design the termination of hybrid undulators and wigglers with reduced field integral variations with gap. Example of such hybrid terminations can be found in [9].

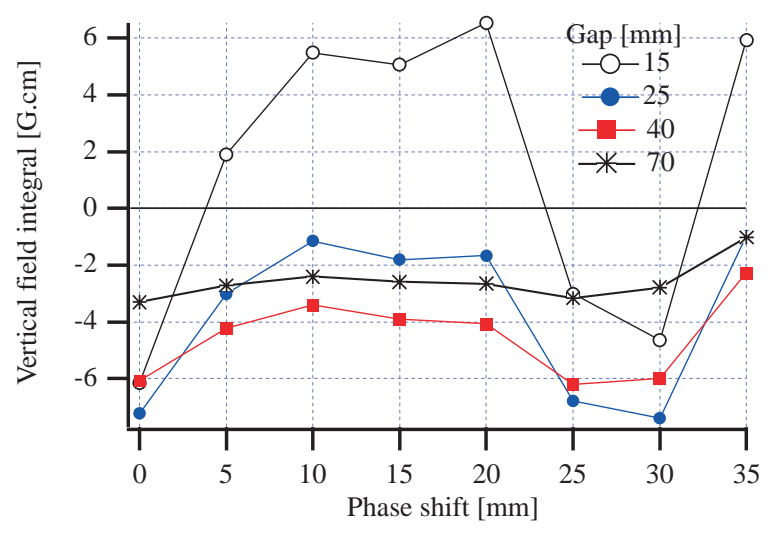

Figure 9: Vertical field integral vs. phase and gap computed for the new termination shown in Figure 7.

\section{REFERENCES}

[1] J. Chavanne, P. Elleaume, Undulator and Wiggler Shimming, Synchrotron Radiation News, Vol. 8, No. 1, 1995, p 18-22

[2] O. Chubar, P. Elleaume, J. Chavanne, "A 3D Magnetostatics Computer Code for Insertion devices", SRI97 Conference August 1997, J. Synchrotron Rad. (1998). 5, 481-484. The Radia code is freely available from "http://www.esrf.fr/machine/support/ids/ Public/Codes/software.html"

[3] S.Sasaki et al., Nucl. Instr. Meth. In Phys. Res. A 331 (1993) 763767.

[4] J. Bahrdt et al., BESSY. A Double Undulator for the Production of Circularly Polarized Light at BESSY II , EPAC' 96 , Barcelone, p. 2538

[5] S. Marks et al., The advanced light source elliptically polarizing undulator. PAC'97, Vancouver, p. 3221

[6] B. Diviacco et al., Construction of elliptical undulators for ELETTRA, EPAC'98, Stockolm, p.2216

[7] Ch. Chang et al., Conceptual design for SRRC elliptically Polarizing Undulator EPU5.6. EPAC' 96, Barcelone, p. 2567

[8] T.Bizen et al., Fabrication and magnetic field measurements of an apple-2 variable polarizing undulator, EPAC'98, Stockholm, p. 2243

[9] J. Chavanne, P. Elleaume, P. Van Vaerenbergh, Segmented High Quality Undulators, Particle Accelerator Conference PAC95, Dallas May 1-5 1995. Proceedings p 1319-1321, IEEE Publication, New Jersey 